\title{
Tumour Expression of Histone Deacetylases in Uveal Melanoma
}

\author{
Louis Levinzon Michele Madigan Vuong Nguyen Enisa Hasic Max Conway \\ Svetlana Cherepanoff \\ Save Site Institute, Sydney Medical School, The University of Sydney, Sydney Eye Hospital, Sydney, NSW, Australia
}

\section{Keywords}

Uveal melanoma · Histone deacetylases · Sirtuins ·

Immunohistochemistry

\section{Abstract}

Purpose: To determine the expression of histone deacetylase enzymes in uveal melanoma tumour cells. Procedures: This is an observational immunohistochemical study of 16 formalin-fixed, paraffin-embedded eyes enucleated for uveal melanoma between January 2001 and March 2002. Haematoxylin and eosin paraffin sections were reviewed for histopathological parameters according to the American Joint Committee on Cancer 7th edition. Sections were then immunohistochemically stained for histone deacetylases 1, 2, 3, 4 and 6 and sirtuin 2 using an automated Leica Bond II platform and Fast Red chromogen, then digitally scanned using Aperio software before assessment of staining. Results: Nuclear expression of histone deacetylases 1, 2, 3, 4 and 6 and of sirtuin 2 was confirmed in uveal melanoma tumour cells. In addition, the tumour cells showed cytoplasmic expression of histone deacetylases 4 and 6 and sirtuin 2. Nuclear and cytoplasmic immunostaining was also seen in intraocular tis-

\section{KARGER}

() 2018 S. Karger AG, Basel

E-Mail karger@karger.com

www.karger.com/oop sues uninvolved by the tumour. Conclusion: Uveal melanoma tumour cells express histone deacetylases 1, 2, 3, 4 and 6 and sirtuin 2, confirming potential tissue targets for histone deacetylase inhibitors.

(c) 2018 S. Karger AG, Basel

\section{Introduction}

Uveal melanoma is the most common primary intraocular tumour in adults, affecting between 6 and 10 people per million [1]. This accounts for between 3 and 5\% of melanoma diagnoses [2], but a disproportionately higher mortality of $13 \%$ [3]. Despite excellent local control [4] and a mere $4 \%$ rate of metastasis at diagnosis [5], 50\% of patients eventually succumb to haematogenous metastases primarily to the liver. These patients have a poor prognosis, with a life expectancy of 6-9 months [6]. Unfortunately, the survival of uveal melanoma patients has remained largely unchanged for nearly three decades [7] and is independent of local tumour control and treatment [8].

Histone deacetylase enzymes (HDACs) have pleiotropic actions affecting cellular differentiation and cancer 
cell behaviour [9]. Abnormal HDAC activity can lead to abnormal gene expression [10] permitting cancer development [11]. Aberrant histone acetylation and HDAC recruitment are implicated in various solid organ tumours and haematological malignancies via effects on chromatin structure and gene expression [10]. Although mutations in HDACs are rare [12], changes in expression have been noted in a number of tumours, and these changes have been associated with malignant behaviour, a higher grade and stage, poorer prognosis and differences in treatment response [13].

The balance between histone acetyltransferases and HDACs determines target protein acetylation and function. Histone proteins are one such target. They control chromatin structure and accessibility to influence transcription, gene replication, recombination and repair [14]. In addition, a diverse group of nuclear and cytoplasmic non-histone proteins are also substrates for histone acetyltransferases and HDACs with diverse roles in DNA transcription [10] and DNA repair, apoptosis, cell metabolism and maintenance of cell structure [15]. Aberration of acetylation is therefore implicated in numerous disease processes including cancer [15], and hypoacetylation is frequently observed in malignancy [16].

There are four classes of HDAC. Class I includes HDACs 1, 2, 3 and 8, which are ubiquitously located in nuclei [17] with generalised roles in cell proliferation and survival [18]. Class II includes HDACs 4, 5, 6, 7, 9 and 10, which are capable of nucleocytoplasmic shuttling [17]. Sirtuins (SIRT) 1-7 compose HDAC class III, and they require nicotinamide adenine dinucleotide $\left(\mathrm{NAD}^{+}\right)$as a co-factor [15]. Sirtuins are variously localised throughout the cell, with SIRT2 located in both the nucleus and cytoplasm [15]. The biological behaviour of class IV HDAC11 is similar to that of both class I and II HDACs [15].

HDAC inhibitors are promising anticancer agents with effects reported in multiple human malignancies and cell lines [19]. These agents display strong selectivity against malignant cells [20], but relatively low toxicity to normal cells [19]. HDAC inhibitors display a broad spectrum of actions targeting the hallmark aberrant behaviours of cancer cells $[9,17]$. The use of HDAC inhibitors in uveal melanoma is supported by HDAC-mediated xenograft cell cycle arrest and by differentiation and growth inhibition in animal models [21]. Class III-specific HDAC inhibitors have similarly shown promise via effects on SIRT1 and SIRT2, inhibiting growth, inducing apoptosis and removing cancer stem cell populations whilst displaying a synergism with conventional chemotherapeutic agents [22].
Clearly, the use of HDAC inhibitors to manage uveal melanoma metastases is both biologically plausible and well supported by in vitro evidence. Despite this, the data from early HDAC inhibitor clinical trials have been disappointing for patients with uveal melanoma [23]. Since HDACs are a diverse group of proteins, we hypothesise that a better understanding of the expression of specific HDACs in human uveal melanoma tissue will lead to a more nuanced and targeted understanding of the roles they play in this devastating disease. Herewith we present immunohistochemical data on the expression of six HDACs (HDAC1, HDAC2, HDAC3, HDAC4, HDAC6 and SIRT2), chosen for their known association with prognosis, grade and stage in other malignancies $[13,24$, 25].

\section{Methods}

\section{Uveal Melanoma Eyes}

All eyes enucleated for uveal melanoma between January 2001 and March 2002 were identified from a database search at South East Area Laboratory Service (SEALS), Prince of Wales Hospital, Sydney, NSW, Australia, with approval from the South Eastern Sydney and Illawarra Area Health Service Human Research Ethics Committee. Archived pathology reports were reviewed for the relevant clinicopathological data that were extracted. Of the 20 eyes identified, 2 were excluded due to tumour necrosis or insufficient residual tumour in the paraffin block, and 2 were excluded due to insufficient data; 16 eyes were examined in this study.

\section{Tissue Fixation, Processing and Staining}

The eyes were fixed in 10\% neutral buffered formalin after enucleation and then embedded in paraffin. Three- to $4-\mu \mathrm{m} \mathrm{sec}-$ tions of the pupil-to-optic nerve block were cut, collected onto slides and stained with haematoxylin and eosin for grading according to the American Joint Committee on Cancer (AJCC) 7th edition. Additional slides for each specimen were prepared for immunohistochemistry using a Leica Bond II automated immunostainer (Leica Microsystems, Norwell, MA, USA) using optimised primary antibody concentrations - HDAC1 (rabbit polyclonal IgG; 1:2,000; Abcam ab-198845), HDAC2 (rabbit polyclonal IgG; 1:500; Santa Cruz sc-7899), HDAC3 (rabbit polyclonal IgG; 1:200; Abcam ab-16047), HDAC4 (rabbit polyclonal IgG; 1: 500; Abcam ab-79521), HDAC6 (mouse monoclonal IgG; 1:500; Santa Cruz sc-28386) and SIRT2 (rabbit polyclonal IgG; 1:800; Santa Cruz sc-20966) - and recommended heat-induced epitope retrieval conditions and IHC Protocol J with a BOND Polymer Define Red detection kit. The slides were coverslipped automatically using Eukitt.

Assessment of Immunohistochemical Staining and Selection of Fields for Quantification

The slides were digitally scanned using an Aperio AT2 Digital Pathology Slide Scanner (MAN-0250, Revision 1; Leica Biosystems Imaging, Inc., Tokyo, Japan) and subsequently viewed using 


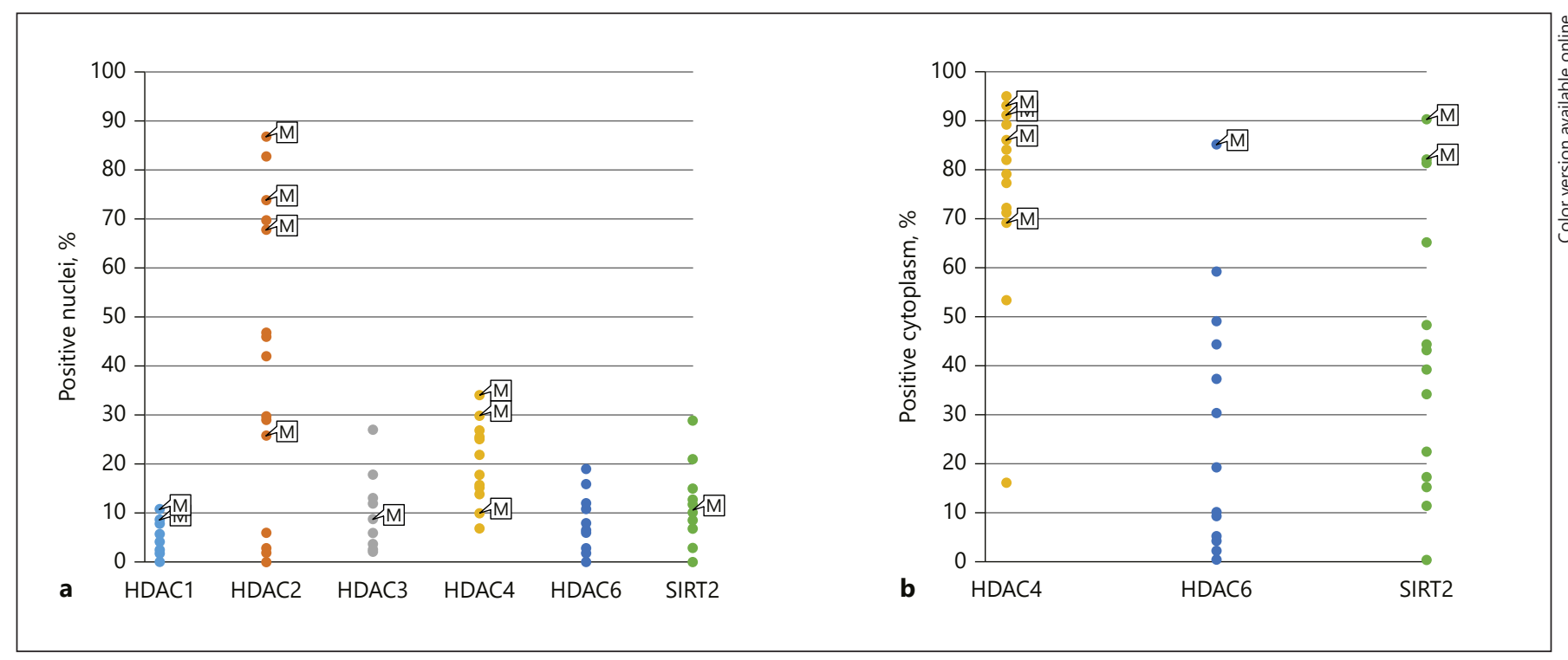

Fig. 1. a Average nuclear staining in percent for HDAC1 (light blue), HDAC2 (orange), HDAC3 (grey), HDAC4 (yellow), HDAC6 (dark blue) and SIRT2 (green) for each eye (noting no result for HDAC1 eye 3, HDAC2 eyes 11 and 12, HDAC6 eye 12, and SIRT2 eyes 10 and 13). The predominant staining was weak, unless specified by "M," indicating that moderate staining pre- dominated. b Average cytoplasmic staining in percent for HDAC4 (yellow), HDAC6 (dark blue) and SIRT2 (green) for each eye (noting no result for HDAC6 eye 12 and SIRT2 eyes 10 and 13). The predominant staining was weak, unless specified by " $M$," indicating that moderate staining predominated. HDAC, histone deacetylase; SIRT, sirtuin.

Table 1. Summary of the demographic and clinicopathologic characteristics of the study eyes

\begin{tabular}{|c|c|c|c|c|c|c|c|c|c|c|c|c|}
\hline Eye No. & $\begin{array}{l}\text { Patient } \\
\text { sex }\end{array}$ & Eye & $\begin{array}{l}\text { Year of } \\
\text { diagnosis }\end{array}$ & $\begin{array}{l}\text { Age at } \\
\text { diagnosis, } \\
\text { years }\end{array}$ & $\begin{array}{l}\text { Extent at } \\
\text { diagnosis }\end{array}$ & $\begin{array}{l}\text { Age at } \\
\text { death, } \\
\text { years }\end{array}$ & $\begin{array}{l}\text { Survival, } \\
\text { years }\end{array}$ & $\begin{array}{l}\text { Tumour } \\
\text { type }\end{array}$ & $\begin{array}{l}\text { Macroscopic } \\
\text { diameter, mm }\end{array}$ & $\begin{array}{l}\text { Macroscopic } \\
\text { thickness, } \mathrm{mm}\end{array}$ & $\begin{array}{l}\text { Extraocular } \\
\text { extension }\end{array}$ & $\begin{array}{l}\text { TNM } \\
\text { stage }\end{array}$ \\
\hline 1 & M & $\mathrm{L}$ & 2001 & 59 & Localised & 61 & 2 & Spindle & 15 & 11 & No & $\mathrm{T} 4$ \\
\hline 3 & $\mathrm{~F}$ & $\mathrm{R}$ & 2001 & 77 & Localised & N/A & $10+$ & Mixed & 11 & 10 & No & $\mathrm{T} 3$ \\
\hline 4 & $\mathrm{M}$ & $\mathrm{R}$ & 2002 & 62 & Localised & $\mathrm{N} / \mathrm{A}$ & $9+$ & Mixed & 13 & 11 & No & $\mathrm{T} 3$ \\
\hline 5 & $\mathrm{~F}$ & $\mathrm{~L}$ & 2001 & 61 & $\mathrm{~N} / \mathrm{S}$ & N/A & $10+$ & Mixed & 9 & 8 & No & $\mathrm{T} 2$ \\
\hline 8 & $\mathrm{~F}$ & $\mathrm{~L}$ & $\mathrm{~N} / \mathrm{S}$ & $\mathrm{N} / \mathrm{S}$ & $\mathrm{N} / \mathrm{S}$ & $\mathrm{N} / \mathrm{S}$ & $\mathrm{N} / \mathrm{S}$ & Mixed & 12 & 7 & No & $\mathrm{T} 3$ \\
\hline 9 & $\mathrm{~F}$ & $\mathrm{~L}$ & $\mathrm{~N} / \mathrm{S}$ & $\mathrm{N} / \mathrm{S}$ & $\mathrm{N} / \mathrm{S}$ & $\mathrm{N} / \mathrm{S}$ & $\mathrm{N} / \mathrm{S}$ & Mixed & 16 & 7 & Yes & $\mathrm{T} 4$ \\
\hline 10 & M & $\mathrm{L}$ & 2002 & 73 & Regionalised & 75 & 2 & Mixed & 17 & 12 & Yes & $\mathrm{T} 4$ \\
\hline 11 & $\mathrm{~F}$ & $\mathrm{R}$ & 2002 & 70 & Localised & 71 & 1 & Mixed & 15 & 9 & No & $\mathrm{T} 3$ \\
\hline 12 & M & $\mathrm{L}$ & 2002 & 43 & Localised & $\mathrm{N} / \mathrm{A}$ & $9+$ & Mixed & 20 & 14 & No & $\mathrm{T} 4$ \\
\hline
\end{tabular}

Survival data were obtained from the Cancer Council of New South Wales. Localised disease: no clinically documented metastases at the time of diagnosis. Regionalised disease: clinically documented metastases at the time of diagnosis. N/A, not applicable (patient alive at the time of the study); N/S, not specified. 

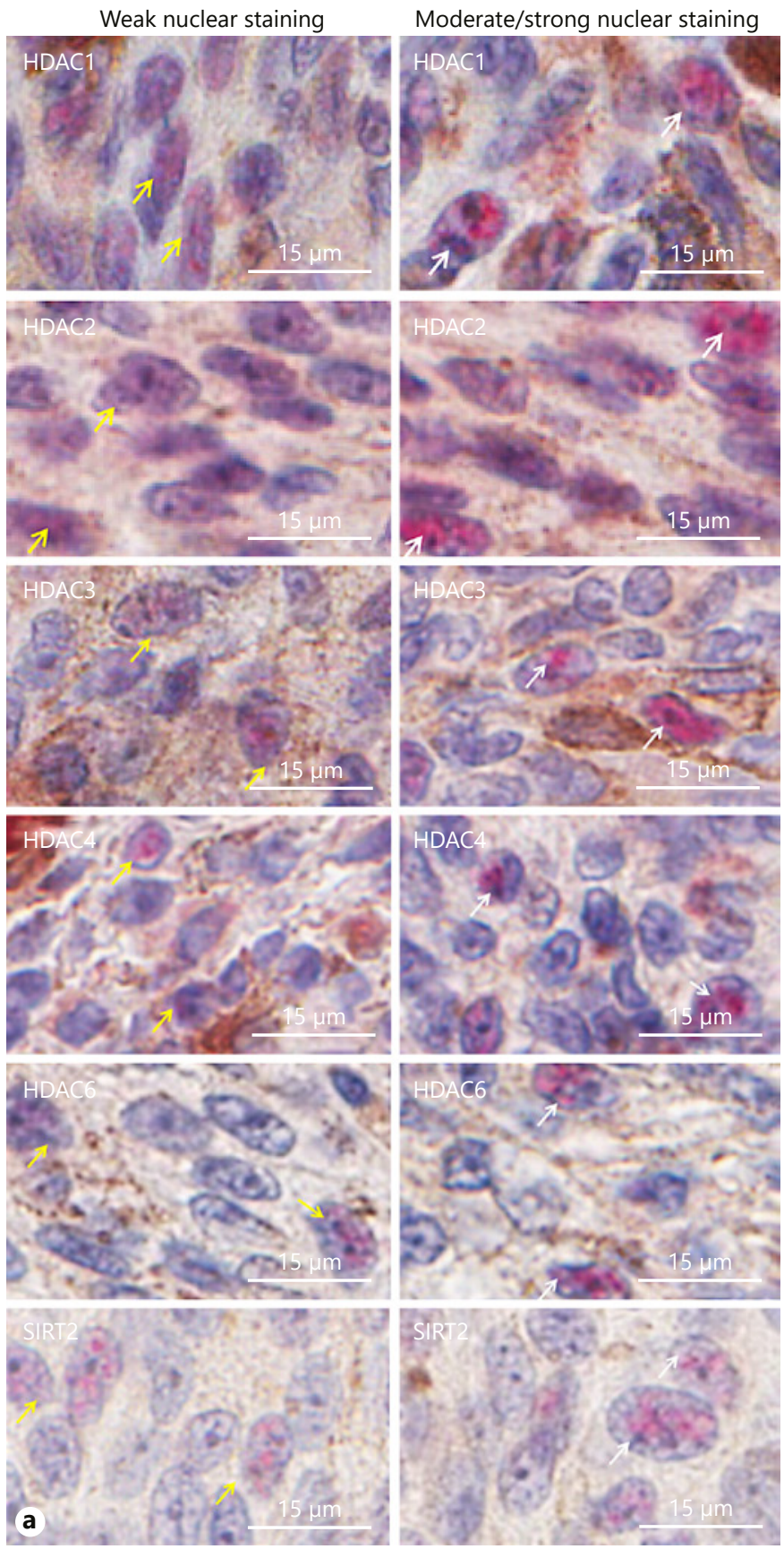

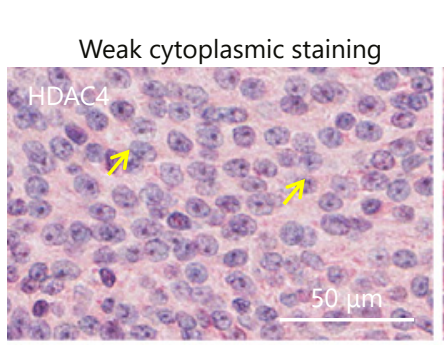

Moderate/strong cytoplasmic staining
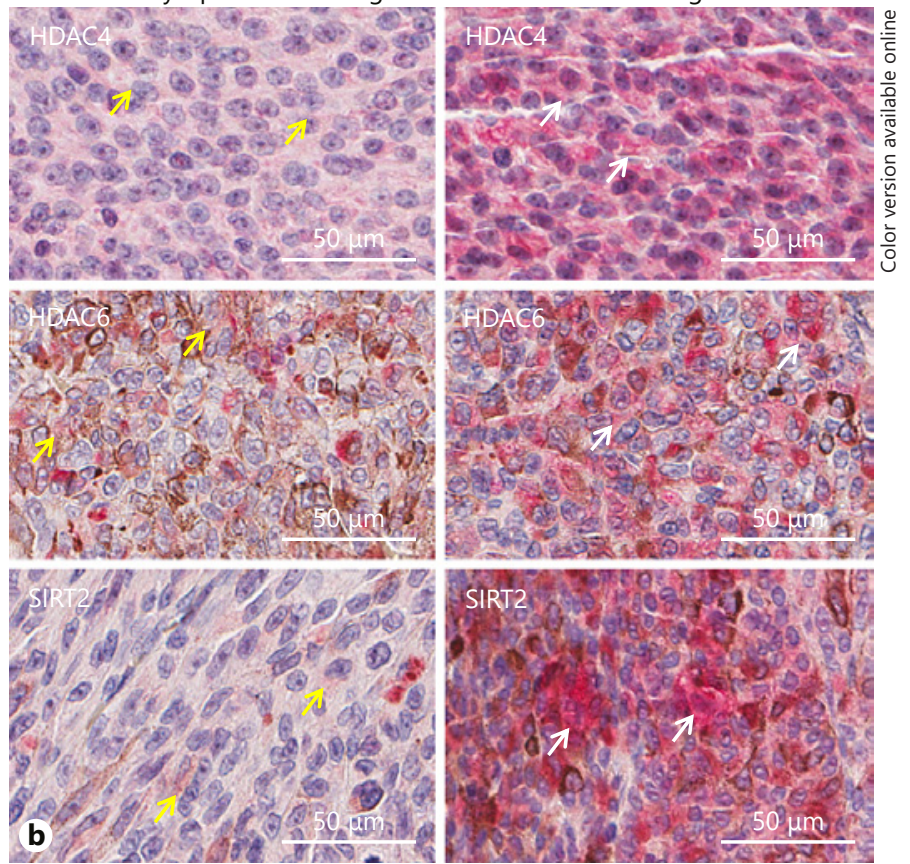

Fig. 2. a Nuclear uveal melanoma tumour expression of HDACs. All uveal melanomas expressed nuclear HDAC1, 2, 3, 4 and 6 and SIRT2. The representative photomicrographs from uveal melanoma tumours show weak (left column, yellow arrows) and moderate/strong (right column, white arrows) nuclear staining in uveal melanoma cells for each HDAC tested. Fast Red chromogen; haematoxylin counterstain. b Cytoplasmic uveal melanoma tumour expression of HDACs. Cytoplasmic staining for HDACs 4 and 6 and SIRT2 was seen in uveal melanoma tumour cells. The representative photomicrographs show weak (left column, yellow arrows) and moderate/strong (right column, white arrows) cytoplasmic staining for HDACs 4 and 6 and SIRT2 in uveal melanoma cells. Fast Red chromogen; haematoxylin counterstain. HDAC, histone deacetylase; SIRT, sirtuin.

ImageScope software (v12.0.1.5027; Aperio Technologies). Nine images could not be assessed due to pixilation and suboptimal immunohistochemistry results - 1 eye for HDAC1, 2 eyes for HDAC2, 2 eyes for HDAC6 and 4 eyes for SIRT2.

For each eye immunostained for HDAC1, 2, 3, 4 and 6 and SIRT2, ten $300 \times 180 \mu \mathrm{m}$ high-power fields (HPF) were selected. The pattern of HDAC staining was first reviewed by 3 observers (S.C., M.C. and M.M.) and consensus criteria were agreed upon. The first author (L.L.) was trained to assess the digitally scanned slides by an anatomical pathologist (S.C.) and research scientist
(M.M.). The staining results were subsequently quantified by $1 \mathrm{ob}-$ server (L.L.) using the consensus staining criteria and ImageScope software at $\times 400$ magnification.

Because tumour-infiltrating macrophages are known to influence the biological behaviour of uveal melanoma, we selected fields for assessment based on the following densities of tumour-infiltrating macrophages: (1) low $-3 \mathrm{HPF}$; (2) moderate $-4 \mathrm{HPF}$; and (3) high - 3 HPF (10 HPF for each eye and each HDAC in total). Macrophages were identified based on their epithelioid morphology, coarse and granular pigmentation, low nuclear-to-cytoplas- 
mic ratio and reniform nuclei [26]. Staining in non-tumour ocular structures was also noted.

The number of tumour cell nuclei staining red was counted for each field, noting the staining intensity (weak or moderate to strong) and then categorised semi-quantitatively into the following groups: $\leq 5,5-24,25-49,50-74$ and $\geq 75 \%$. The tumour cell's cytoplasmic staining intensity was noted (weak or moderate to strong) and the cytoplasmic area stained was approximated semiquantitatively into the following groups: $\leq 5,5-24,25-49,50-74$ and $\geq 75 \%$. The predominant staining intensity for each eye and the average percentage of positive nuclei (Fig. 1a) and cytoplasmic area (Fig. 1b) for 10 regions was noted and summarised into scatter plots.

\section{Results}

The characteristics of the study eyes are summarised in Table 1 . The majority of the study eyes (94\%) were AJCC pT3 or greater and most had a mixed epithelioid/ spindle histology.

\section{Immunohistochemistry}

All uveal melanoma cases in the study expressed HDACs 1, 2, 3, 4 and 6 and SIRT2. Staining was nuclear and predominantly weak and granular. Figure 1a summarises the predominant nuclear staining intensity for each eye and the average percentage of positive cells for 10 regions. HDAC2 showed the strongest nuclear staining, with diffuse moderate-to-strong staining observed in $>75 \%$ of tumour cells per HPF. Examples of weak and moderate-to-strong nuclear staining for all HDACs are shown in Figure 2a.

In addition, we found that three HDACs - HDAC4, HDAC6 and SIRT2 - were expressed in the cytoplasm of uveal melanoma tumour cells. Figure $1 \mathrm{~b}$ summarises the predominant cytoplasmic staining intensity for each eye and the average percentage of positive cells for 10 regions. Staining was predominantly weak and diffuse; however, HDAC4 had the strongest and most diffuse staining, which often exceeded the intensity of its nuclear staining. Examples of weak and moderate-to-strong cytoplasmic staining for all HDACs are shown in Figure $2 \mathrm{~b}$.

Online supplementary Figure 1 (for all online suppl. material, see www.karger.com/doi/10.1159/000490038) demonstrates the overall HDAC staining with insets of uveal melanocytes as a control.

We also observed nuclear and cytoplasmic HDAC immunostaining in non-tumour ocular structures including the lens, optic nerve head and retina in the 16 eyes examined (Fig. 3). These qualitative observations are summarised in Table 2.

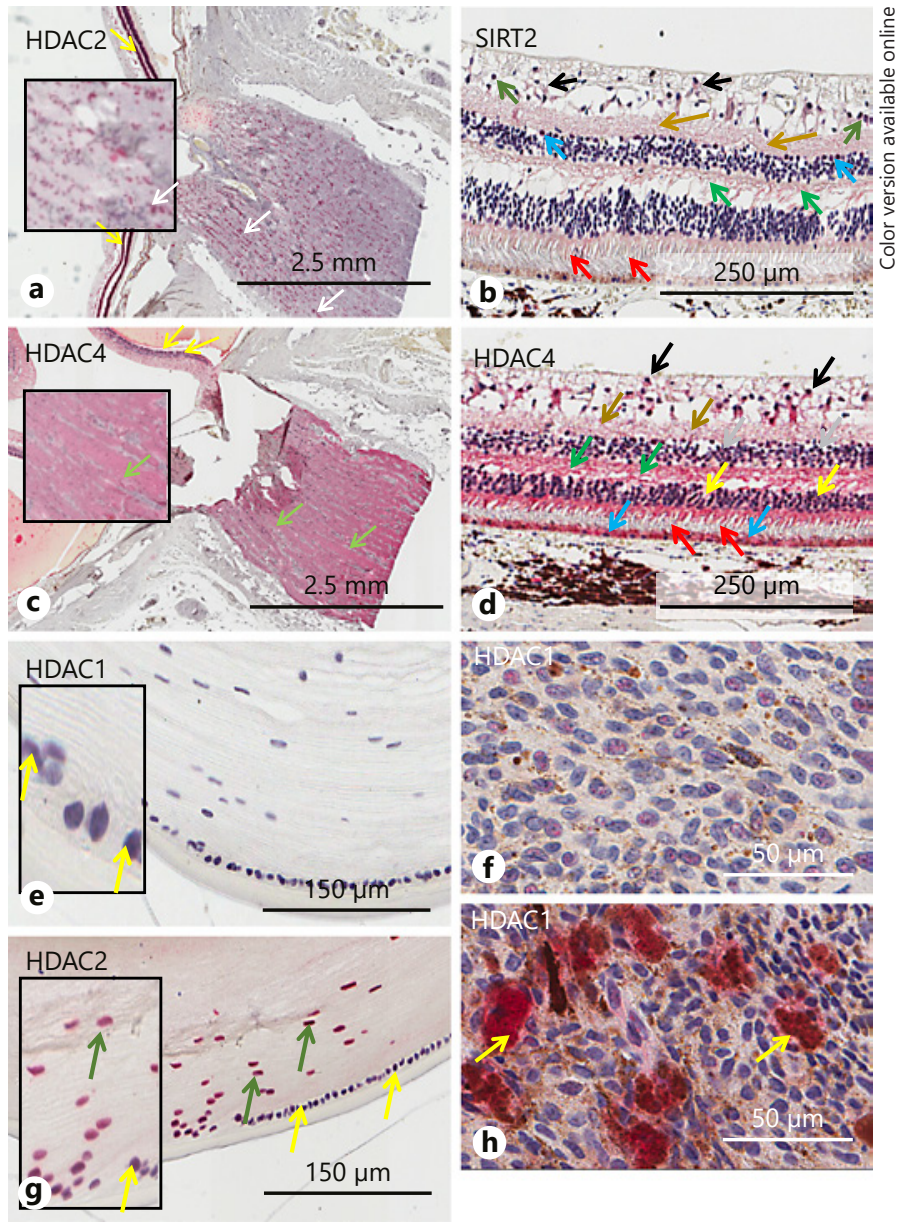

Fig. 3. Photomicrographs of HDAC staining in normal ocular tissues. a, c Optic nerve head, demonstrating positive HDAC2 staining of the inner and outer nuclear layers of the retina (a; yellow arrows) and optic nerve glia (a; white arrows) as well as positive HDAC4 staining of optic nerve cytoplasm (c; green arrows) and the retina (c; yellow arrows), further visualised in $\mathbf{d}$. $\mathbf{d}$ Cytoplasmic HDAC4 staining of ganglion cells (black arrows) and the inner and outer nuclear layers, as well as cytoplasmic staining of the ganglion cell layer, the inner (brown arrows) and outer (green arrows) plexiform layers, the inner (grey arrows) and outer (yellow arrows) nuclear layers, photoreceptors (red arrows) and the retinal pigment epithelium (blue arrows). b Cytoplasmic SIRT2 staining of the ganglion cell layer (black arrows), as well as nuclear staining of ganglion cells (aqua arrows), the inner (brown arrows) and outer (green arrows) plexiform layers, and photoreceptors (red arrows); some nuclear staining was noted in the inner nuclear layer (light blue arrows). e, g Weak nuclear HDAC1 staining of lens epithelial cells at the lens bow (e; yellow arrows) and weak nuclear HDAC2 staining of lens epithelial cells (g; yellow arrows) and strong nuclear HDAC2 staining of lens fibre cells (g; green arrows). f HDAC1 demonstrating a low-macrophage-density region. $\mathbf{h} \mathrm{HDAC1}$ demonstrating a high-macrophage-density region (yellow arrows). HDAC, histone deacetylase; SIRT, sirtuin. 
Table 2. Summary of nuclear and cytoplasmic immunostaining observed for HDAC1, HDAC2, HDAC3, HDAC4, HDAC6 and SIRT2 in non-tumour ocular structures

\begin{tabular}{|c|c|c|c|c|c|c|}
\hline Structure & HDAC1 & HDAC2 & HDAC3 & HDAC4 & HDAC6 & SIRT2 \\
\hline \multicolumn{7}{|l|}{ Nuclear } \\
\hline Ciliary body pigmented epithelium & $\mathrm{x}$ & $\mathrm{x}$ & $\mathrm{x}$ & $\mathrm{x}$ & $\mathrm{x}$ & $\mathrm{x}$ \\
\hline Ciliary body non-pigmented epithelium & & $\mathrm{x}$ & $\mathrm{x}$ & $\mathrm{x}$ & & \\
\hline Ciliary muscle & & & & $\mathrm{x}$ & & \\
\hline Posterior iris pigment epithelium & & & & $\mathrm{x}$ & & \\
\hline Iris dilator muscle & & & & $\mathrm{x}$ & & \\
\hline Optic nerve head axons & & $\mathrm{x}$ & $\mathrm{x}$ & $\mathrm{x}$ & & $\mathrm{x}$ \\
\hline $\begin{array}{l}\text { Hyalinised Bruch's membrane and } \\
\text { choriocapillaris }\end{array}$ & & & $\mathrm{x}$ & & & \\
\hline Retinal pigment epithelium & & & & $\mathrm{x}$ & & $\mathrm{x}$ \\
\hline Inner and outer segments & & & & $\mathrm{x}$ & $\mathrm{x}$ & $\mathrm{x}$ \\
\hline Outer plexiform layer & & & $\mathrm{x}$ & $\mathrm{x}$ & & $\mathrm{x}$ \\
\hline Inner plexiform layer & & & $\mathrm{x}$ & $\mathrm{x}$ & & $\mathrm{x}$ \\
\hline Nerve fibre layer & & & $\mathrm{x}$ & $\mathrm{x}$ & & $\mathrm{x}$ \\
\hline Corneal epithelium & & & & $\mathrm{x}$ & & \\
\hline Lens epithelium & & & & $\mathrm{x}$ & & $\mathrm{x}$ \\
\hline Lens fibre cells & & & & $\mathrm{x}$ & & $\mathrm{x}$ \\
\hline $\begin{array}{l}\text { Cytoplasmic } \\
\text { Ciliary muscle }\end{array}$ & & $\mathrm{x}$ & & & & \\
\hline Optic nerve head glia & & $\mathrm{x}$ & $\mathrm{x}$ & & & \\
\hline Outer nuclear layer & & $\mathrm{x}$ & $\mathrm{x}$ & $\mathrm{x}$ & & \\
\hline Inner nuclear layer & & $\mathrm{x}$ & $\mathrm{x}$ & $\mathrm{x}$ & & $\mathrm{x}$ \\
\hline Ganglion cell layer & & $\mathrm{x}$ & & $\mathrm{x}$ & & $\mathrm{x}$ \\
\hline Corneal epithelium & & & & $\mathrm{x}$ & & \\
\hline Lens epithelium & $\mathrm{x}$ & $\mathrm{x}$ & $\mathrm{x}$ & & & \\
\hline Lens fibre cells & & $\mathrm{x}$ & & $\mathrm{x}$ & & \\
\hline
\end{tabular}

\section{Discussion}

This study provides the first evidence of tissue expression of several classes of HDAC (HDACs 1, 2, 3, 4 and 6 and SIRT2) in both uveal melanoma nuclei and cytoplasm. The observed nuclear staining of all HDACs examined is consistent with their known nuclear localisation [27]. The predominance of strong nuclear staining for class IHDACs, especially HDAC2 and HDAC3, is in keeping with the gen- eral upregulation of class I HDACs in malignancy [13]. Class I HDACs are reported to be upregulated in malignancies [13]. HDAC2 is upregulated and linked to prognosis in cancers including gynaecological and haematological malignancies [13] and is reported to have a definitive role in the development of colorectal carcinoma facilitating tumorigenesis in a mouse model with catalytically inactive HDAC2 and APC mutation [28]. Class II HDACs, on the other hand, are generally downregulated in malignancy 
[13]. Low-level HDAC6 staining in uveal melanoma cells was observed in this study.

The nuclear and cytoplasmic staining of HDAC4, HDAC6 and SIRT2 is in keeping with known patterns of partitioning of these HDACs [15, 27]. HDAC4 overexpression is generally associated with an improved prognosis, as noted in HER2-positive and luminal B breast cancers [13]. Specific targeting of HDAC4 may also inhibit HIF-1 $\alpha$-dependent inflammatory responses [29]. We observed that HDAC4 expression was strongest for the cytoplasmic area and intensity in uveal melanoma. HDAC4 is often upregulated in malignancies with excess inflammation and chemokine signalling [30], consistent with an inflammatory phenotype known to enhance the progression of uveal melanoma [31]. In some immune cells, controlled nuclear export of HDAC4 plays an important role in cytokine production [28]. As a result, elevated cytosolic levels may have a role in the nuclear gene expression of inflammatory targets or cytoplasmic nonhistone protein function that enhances malignant behaviour. Further studies in uveal melanoma to examine the effects of inflammatory signalling and key acetylated proteins, activity and location are required to determine whether tumour-promoting signals may allow for phosphorylation of HDAC4 sequestering protein 14-3-3 to release HDAC4 and allow it to shuttle to the nucleus to affect gene expression [10].

HDAC6 and SIRT2 are $\alpha$-tubulin deacetylases that function to reduce the stability of $\alpha$-tubulin and thus promote cell motility and metastasis [32]. A recent study $(n=21)$ demonstrated significantly higher SIRT2 immunostaining in uveal melanoma cells compared to normal melanocytes, suggesting a role as an oncogene and therapeutic target [33]. However, in our study $(n=14)$, we noticed a range of staining of uveal melanoma cells for HDAC6 and SIRT2. This may be related to variability in the study specimens. The role of SIRT2 in the motility and metastasis of uveal melanoma requires further study.

Our study also noted novel HDAC staining in nontumour ocular structures. We observed nuclear and cytoplasmic staining of HDAC2 and HDAC 3 in the axons and glia of the optic nerve head; other studies have previously noted staining with these HDACs in the human lamina cribrosa beams and fibroblasts of the peripapillary sclera [34].

SIRT2 has been shown to be expressed in a human retinal pigment epithelial cell line [35], with enzymatic activity of several HDACs including HDACs 1, 2, 3 and 6 also detected in cell culture [36]; however, this study has demonstrated immunohistochemical expression of

HDAC Expression in Uveal Melanoma
HDAC4 and SIRT2 in the retinal pigment epithelium. SIRT2 has also been detected in the non-pigmented ciliary body epithelium [25], but this was not noted in this study.

Although one study demonstrated HDAC1, 2, 3, 5, 6 and 7 proteins in human retinal supernatant via mass spectrometry [37] and another study demonstrated SIRT2 in the inner and outer nuclear and plexiform layers and nerve fibre layer [25], we did not note consistent HDAC1 staining in the retina, instead noting widespread staining of HDAC4 and SIRT2 followed by HDACs 2, 3 and 6.

HDAC1 [38] and HDAC6 [39] proteins have previously been detected in the corneal epithelium; however, this study did not observe this, instead noting HDAC4 staining in the basal layers of the corneal epithelium. HDAC1 protein has also been noted in the human lens capsule [40], but this was not observed, instead noting HDAC4 and SIRT2 staining of lens epithelial and fibre cells.

Our observations demonstrate HDAC expression in uveal melanoma that may be amenable to pharmacological inhibition. The HDAC inhibitors vorinostat, romidepsin and belinostat are currently approved for the treatment of relapsed or resistant cutaneous and peripheral T-cell lymphomas [24]. In solid organ malignancies, these agents are less effective as monotherapy [24]; currently, numerous clinical trials for other haematological and solid organ malignancies [41] are using HDAC inhibitors combined with other chemotherapeutic agents [42]. In some tumours, a strong level of HDAC expression is suspected to be indicative of a positive response to HDAC inhibition [24]. Two phase II clinical trials are currently investigating vorinostat in metastatic uveal melanoma [43] and one trial is investigating adjuvant valproic acid in the prevention of metastatic disease [44]. HDAC inhibitors are expected to sensitise cancer cells to chemotherapy and radiotherapy [18]. For example, a phase I clinical trial has reported an uncharacteristic sensitivity of cutaneous melanoma to doxorubicin, producing stable disease, where HDAC2 was predictive of response [45]. HDAC inhibitors are also suspected to induce differentiation and maintain quiescence of micrometastatic disease [46], further supporting an adjuvant role for these agents in treating uveal melanoma.

Our sample size was small ( $n=16$ eyes). Correlation with clinical follow-up and survival data in a larger sample is necessary to determine whether HDAC expression is associated with poor prognosis or metastasis. There is an inherent bias in this study, as enucleated eyes with more advanced disease were included rather than eyes with smaller, early-stage tumours that are treated with 
brachytherapy and not usually enucleated until progression occurs.

In future studies, a complete panel of all 18 HDACs will be examined for primary and metastatic melanoma at both the mRNA and the protein level. Combined with correlation to known prognostic factors derived from a dedicated uveal melanoma registry, this will further enhance our understanding of the involvement of HDACs in melanoma pathobiology and inform the development of specific HDAC inhibitors for therapy.

Knowledge of the specific HDAC isoforms involved in the development of uveal melanoma will allow for more specific HDAC inhibitors with better tolerability. This may either minimise the risk of micrometastases at early stages or induce differentiation of macrometastases in advanced disease in the hope of improving prognosis [46].

Despite these limitations, we did find distinct evidence of nuclear and cytoplasmic expression patterns of several classes of HDACs in uveal melanoma, providing histological evidence of a possible target for the use of HDAC inhibitors in therapy.

\section{Acknowledgements}

We would like to thank the National Foundation for Medical Research and Innovation for ongoing funding.

\section{Statement of Ethics}

The South Eastern Sydney and Illawarra Area Health Service Human Research Ethics Committee approved this study.

\section{Disclosure Statement}

The authors have no conflicts of interest to declare.

\section{Funding Sources}

National Foundation for Medical Research and Innovation and the Claffy Foundation.

\section{References}

1 Griewank KG, Murali R: Pathology and genetics of uveal melanoma. Pathology 2013;45: $18-27$.

2 Patel SP: Latest developments in the biology and management of uveal melanoma. Curr Oncol Rep 2013;15:509-516.

3 Harbour JW: The genetics of uveal melanoma: an emerging framework for targeted therapy. Pigment Cell Melanoma Res 2012;25: 171-181.

4 Harbour JW: Update in uveal melanoma. Clin Adv Hematol Oncol 2012;10:459-461.

5 Jovanovic P, Mihajlovic M, Djordjevic-Jocic J, Vlajkovic S, Cekic S, Stefanovic V: Ocular melanoma: an overview of the current status. Int J Clin Exp Pathol 2013;6:1230-1244.

6 Woodman SE: BAP1tism of a tumor suppressor. Clin Cancer Res 2012;18:323-325.

7 Logan PT, Fernandes BF, Di Cesare S, Marshall JC, Maloney SC, Burnier MN Jr: Singlecell tumor dormancy model of uveal melanoma. Clin Exp Metastasis 2008;25:509-516.

8 Choudhary MM, Triozzi PL, Singh AD: Uveal melanoma: evidence for adjuvant therapy. Int Ophthalmol Clin 2015;55:45-51.

9 Munshi A, Tanaka T, Hobbs ML, Tucker SL, Richon VM, Meyn RE: Vorinostat, a histone deacetylase inhibitor, enhances the response of human tumor cells to ionizing radiation through prolongation of $\gamma$-H2AX foci. Mol Cancer Ther 2006;5:1967-1974.

10 Verdin E: Histone Deacetylases: Transcriptional Regulation and Other Cellular Functions. Totowa, Humana Press, 2006.
11 Li Z, Zhu WG: Targeting histone deacetylases for cancer therapy: from molecular mechanisms to clinical implications. Int J Biol Sci 2014; 10:757-770

12 He S, Li X, Chan N, Hinton DR: Review: epigenetic mechanisms in ocular disease. Mol Vis 2013;19:665-674.

13 Weichert W: HDAC expression and clinical prognosis in human malignancies. Cancer Lett 2009;280:168-176.

14 Herlihy N, Dogrusöz M, van Essen TH, Harbour JW, van der Velden PA, van Eggermond $\mathrm{MC}$, et al: Skewed expression of the genes encoding epigenetic modifiers in high-risk uveal melanoma. Invest Ophthalmol Vis Sci 2015;56:1447-1458.

15 Yao T-P, Seto E: Histone Deacetylases: The Biology and Clinical Implication. Heidelberg, Springer, 2011.

16 Lai F, Jin L, Gallagher S, Mijatov B, Zhang XD, Hersey P: Histone deacetylases (HDACs) as mediators of resistance to apoptosis in melanoma and as targets for combination therapy with selective BRAF inhibitors. Adv Pharmacol 2012;65:27-43.

17 Mottet D, Castronovo V: Histone deacetylases: target enzymes for cancer therapy. Clin Exp Metastasis 2008;25:183-189.

18 Dokmanovic M, Clarke C, Marks PA: Histone deacetylase inhibitors: overview and perspectives. Mol Cancer Res 2007;5:981-989.
19 Venza I, Visalli M, Oteri R, Cucinotta M, Teti D, Venza M: Class II-specific histone deacetylase inhibitors MC1568 and MC1575 suppress IL-8 expression in human melanoma cells. Pigment Cell Melanoma Res 2013;26:193-204.

20 Schwabe M, Lübbert M: Epigenetic lesions in malignant melanoma. Curr Pharm Biotechnol 2007;8:382-387.

21 Landreville S, Agapova OA, Matatall KA, Kneass ZT, Onken MD, Lee RS, et al: Histone deacetylase inhibitors induce growth arrest and differentiation in uveal melanoma. Clin Cancer Res 2012;18:408-416.

22 Dai W, Zhou J, Jin B, Pan J: Class III-specific HDAC inhibitor Tenovin-6 induces apoptosis, suppresses migration and eliminates cancer stem cells in uveal melanoma. Sci Rep 2016;6:22622.

23 Haas NB, Quirt I, Hotte S, McWhirter E, Polintan R, Litwin S, et al: Phase II trial of vorinostat in advanced melanoma. Invest New Drugs 2014;32:526-534.

24 Chun P: Histone deacetylase inhibitors in hematological malignancies and solid tumors. Arch Pharm Res 2015;38:933-949.

25 Orellana ME, Quezada C, Maloney SC, Antecka E, Balazsi M, Burnier MN Jr: Expression of SIRT2 and SIRT6 in retinoblastoma. Ophthalmic Res 2015;53:100-108.

26 Biscotti CV, Singh AD: FNA Cytology of Ophthalmic Tumors. Basel, Karger, 2012.

27 Mottet D, Castronovo V: Histone deacetylases: target enzymes for cancer therapy. Clin Exp Metastasis 2008;25:183-189. 
28 Falkenberg KJ, Johnstone RW: Histone deacetylases and their inhibitors in cancer, neurological diseases and immune disorders. Nat Rev Drug Discov 2014;13:673-691.

29 Shakespear MR, Halili MA, Irvine KM, Fairlie DP, Sweet MJ: Histone deacetylases as regulators of inflammation and immunity. Trends Immunol 2011;32:335-343.

30 Cohen AL, Piccolo SR, Cheng L, Soldi R, Han B, Johnson WE, et al: Genomic pathway analysis reveals that EZH2 and HDAC4 represent mutually exclusive epigenetic pathways across human cancers. BMC Med Genomics 2013;6:35.

31 Bronkhorst IH, Jager MJ: Inflammation in uveal melanoma. Eye (Lond) 2013;27:217223.

32 Aldana-Masangkay GI, Sakamoto KM: The role of HDAC6 in cancer. J Biomed Biotechnol 2011;2011:875824.

33 Halfed DG, Zoroquiain P, Wood HA, Blanco P, Al-Saati N, Aldrees S, et al: SIRT2 expression is higher in uveal melanoma than in ocular melanocytes. Ocul Oncol Pathol 2015;2: 100-104.

34 Park HL, Kim JH, Jung Y, Park CK: Racial differences in the extracellular matrix and histone acetylation of the lamina cribrosa and peripapillary sclera. Invest Ophthalmol Vis Sci 2017;58:4143-4154.
35 Meléndez García R, Arredondo Zamarripa D, Arnold E, Ruiz-Herrera X, Noguez Imm R, Baeza Cruz G, et al: Prolactin protects retinal pigment epithelium by inhibiting sirtuin 2-dependent cell death. EBioMedicine 2016; 7:35-49.

36 Thangaraju M, Karunakaran SK, Itagaki S, Gopal E, Elangovan S, Prasad PD, et al: Transport by SLC5A8 with subsequent inhibition of histone deacetylase 1 (HDAC1) and HDAC3 underlies the antitumor activity of 3-bromopyruvate. Cancer 2009;115:4655-4666.

37 Anderson KW, Chen J, Wang M, Mast N, Pikuleva IA, Turko IV: Quantification of histone deacetylase isoforms in human frontal cortex, human retina, and mouse brain. PLoS One 2015;10:e0126592.

38 Macaluso M, Montanari M, Marshall CM, Gambone AJ, Tosi GM, Giordano A, et al: $\mathrm{Cy}$ toplasmic and nuclear interaction between $\mathrm{Rb}$ family proteins and PAI-2: a physiological crosstalk in human corneal and conjunctival epithelial cells. Cell Death Differ 2006;13: 1515-1522.

39 Wang J, Lin A, Lu L: Effect of EGF-induced HDAC6 activation on corneal epithelial wound healing. Invest Ophthalmol Vis Sci 2010;51:2943-2948.
40 Wang Y, Li F, Zhang G, Kang L, Guan H: Ultraviolet-B induces ERCC6 repression in lens epithelium cells of age-related nuclear cataract through coordinated DNA hypermethylation and histone deacetylation. Clin Epigenetics 2016;8:62

41 Shan X, Fu YS, Aziz F, Wang XQ, Yan Q, Liu JW: Ginsenoside Rg3 inhibits melanoma cell proliferation through down-regulation of histone deacetylase 3 (HDAC3) and increase of p53 acetylation. PLoS One 2014;9:e115401.

42 Arrighetti N, Corno C, Gatti L: Drug combinations with HDAC inhibitors in antitumor therapy. Crit Rev Oncog 2015;20:83-117.

43 Harbour JW, Chao DL: A molecular revolution in uveal melanoma: implications for patient care and targeted therapy. Ophthalmology 2014;121:1281-1288.

44 Amirouchene-Angelozzi N, Schoumacher M, Stern MH, Cassoux N, Desjardins L, PipernoNeumann S, et al: Upcoming translational challenges for uveal melanoma. Br J Cancer 2015;113:1249-1253

45 Munster PN, Marchion D, Thomas S, Egorin M, Minton S, Springett G, et al: Phase I trial of vorinostat and doxorubicin in solid tumours: histone deacetylase 2 expression as a predictive marker. Br J Cancer 2009;101:1044-1050

46 Shields JA: Management of posterior uveal melanoma: past, present, future. Retina 2002; $22: 139-142$. 